\title{
CONSTRUYENDO LOS ROLES PARENTALES EN EL SENO DE LAS DINÁMICAS ASISTENCIALES INSTITUCIONALES
}

\author{
José Manuel HERNÁNDEZ GARRE'; Paloma ECHEVARRÍA PÉREZ'; \\ María José GOMARIZ SANDOVAL ${ }^{2}$ \\ Universidad Católica de Murcia ${ }^{1}$. Hospital Virgen de la Arrixaca ${ }^{2}$ \\ jmhernandez@ucam.edu,pechevarria@ucam.edu,jmgomariz@hotmail.com
}

\section{BUILDING PARENTAL ROLES WITHIN THE DYNAMICS OF INSTITUTIONAL CARE}

Resumen: El primer contacto con el rol parental tiene lugar tras el parto, proceso que en las sociedades industriales se instituye en el marco de una serie de dinámicas clínicas productivistas características de los habitáculos hospitalarios contemporáneos. En este «habitus» se van conformando las primeras atribuciones ligadas a los roles paternales, dibujadas sobre el fondo de una serie de claro-oscuros surgidos tras los duros rituales iniciáticos propios de los paritorios «cosmopolitas». Se trata de una construcción cultural de los roles parentales, que se ha querido etnografíar en el presente artículo desde la óptica de los propios padres. Para ello se ha realizado un análisis del discurso «emic» que, partiendo de un posicionamiento teórico postmoderno, reflexiona sobre el alcance de las dinámicas clínicas hegemónicas y su implicación en las experiencias ligadas a la maternidad/paternidad.

Abstract: The first contact with the parental role occurs after childbirth, process in industrial societies is instituted as part of a series of dynamic productivist clinical characteristics of contemporary hospitable habitats. In this "habitus" are shaped early parental responsibilities related to roles, drawn on the bottom of a series of chiaroscuro emerged after the tough initiatory rituals own of the "cosmopolitan" delivery rooms. It is a cultural construct of parental roles, which wanted ethnography in this article from the perspective of the parents themselves. For this it has made a discourse analysis 'emic' which, from a postmodern theoretical position, reflects on the scope of the hegemonic dynamics and involvement in clinical experiences related to maternity/paternity.

Palabras clave: Roles sociales. Maternidad. Paternidad. Puerperio clínico Social roles. Maternity. Paternity. Clinical postpartum 


\section{Introducción}

El ser padres es un hecho biológico y a la vez cultural en el que surgen las paradojas propias de la interacción entre estas dos dimensiones (Ranisio, 1998). Lo que está en juego es la adopción de diversas formas de identidad (Comas d`Argenir, 1996), de nuevos roles construidos desde la biología pero alimentados por determinantes culturales y étnicos (Jordan, 1993) como la religión, la política, las costumbres, la tradición o la economía (Small, 1999). El primer contacto con este nuevo papel parental se produce, en las sociedades occidentales, en el marco de unas dinámicas hospitalarias que dictan el control puerperal de las madres durante los primeros días posparto. En este sentido, las atribuciones iniciáticas del nuevo estatus social se estructuran durante la reclusión institucional típica de los partos hospitalarios, es decir, en el seno de unas dinámicas asistenciales caracterizadas por atributos como la medicalización, el control biotecnológico, la protocolización, la fragmentación del cuidado o el monopolio médico (Hernández y Echevarría, 2014; Pizzini, 1989; Stoller, 1974). En el «habitus» de esta realidad clínica, y enmarcados sobre los claro-oscuros de las dos actividades típicas del puerperio, la recuperación física y la crianza del bebé (Hernández, 2011; Blázquez, 2009), se producen los primeros intentos de ser padres. Se trata de un momento crítico en el que, a pesar de las molestias derivadas del duro ritual vivido en paritorio, hay que hacer frente al nuevo rol de padres, surgiendo así los conflictos entre las necesidades biológicas, la propia dinámica asistencial y las presión sociales que defienden la implantación de determinados idearios parentales colectivos. En este escenario de la procreación se dirimen las principales representaciones de la nueva paternidad, contexto en el que los modelos de crianza se instituyen en parte nuclear del nuevo dilema existencial de los padres. En este sentido, las representaciones culturales son diversas y van desde los patrones de apego a los distantes, prefiguraciones en cuya trama se arbitran cuestiones esenciales como el tipo de alimentación, los patrones de sueño o la respuesta a los llantos del bebé que, más allá de las necesidades biológicas, son también moduladas por las cosmovisiones sociales e históricas hegemónicas (Small, 1999; Rodríguez, 2015). En el desempeño de estas nuevas atribuciones los padres se distribuyen las primeras labores de crianza, reparto tamizado por las necesidades biológicas pero también por representaciones e ideologías de género preexistentes.

Estamos, en definitiva, ante la construcción individual y social de un nuevo rol, el de padres, que empieza a vertebrarse en el seno de las dinámicas asistenciales propias del puerperio hospitalario. Fenómeno que se ha pretendido investigar en el presente estudio, con el objetivo de relatar, desde la propia óptica de los padres, los principales conflictos y paradojas que surgen en el curso de esta asunción del nuevo rol parental. En este sentido, el análisis se ha realizado desde un posicionamiento teórico postmoderno ${ }^{1}$ que, partiendo de cierta perspectiva de género², reflexiona sobre las dinámicas clínicas dominantes y sus implicaciones en las experiencias ligadas a la maternidad/paternidad.

\footnotetext{
1 Se trata de un posicionamiento encarnado por autores como Gilles Deleuze, Jean-François Lyotard, Michel Foucault o Jacques Derrida desde el que se cuestionan las verdades absolutas defendidas desde las ideologías modernistas, y se piensan que la certeza no es más que una verdad relativamente interpretada y por lo tanto incierta. Perspectiva desde la que se cuestiona el quehacer de las diferentes instituciones sociales y políticas. 2 Estamos ante un movimiento ideológico iniciado por autores como Margaret Mead, Simone Beavouir o John Money, que reflexiona sobre todas las representaciones culturales y sociales que construyen la identidad masculina y femenina. El objetivo es examinar el impacto del sexo en las oportunidades de las personas, sus roles sociales y las interacciones mutuas.
} 


\section{Metodología}

Para el desarrollo de la investigación se optó por un enfoque cualitativo que permitiera recoger los testimonios parentales desde la propia perspectiva «emic». Se partió de la premisa de que los padres tienen su propio punto de vista, siendo el análisis del discurso la mejor estrategia metodológica para recoger los testimonios clave acerca de cómo los nuevos padres se percibían a sí mismos y a la realidad que les rodeaba (Menéndez 2000). El objetivo del enfoque ha sido obtener los datos e interpretaciones más adecuadas (Menénedez y Di Pardo, 1996), caracterizando así "con la mayor precisión posible las representaciones, experiencias y prácticas sociales" (Osorio 2001: 43) de los iniciados al nuevo rol parental. Pese a ello, se dejan ver las inevitables limitaciones que surgen al abordar un fenómeno de estudio desde una «individualidad tipo» que, aunque sea una expresión ideal de la realidad a etnografiar, también presenta cierto grado de aleatoriedad. En este sentido se ha tenido en cuenta que las relaciones durante la investigación no son nunca totalmente neutras ni simétricas, sino que responden a ciertos posicionamientos teóricos y metodológicos adoptados por los implicados en la investigación, entre ellos los propios investigadores (Romani, 1992). De ello deviene que los resultados, a pesar de reflejar las atribuciones de rol parental más comunes en los puerperios hospitalarios, no pueden ser totalmente categóricos ni extrapolables a todas las madres y padres, sino el reflejo de una convección social mayoritaria.

La técnica de investigación utilizada fueron las entrevistas en profundidad semiestructuradas a mujeres y hombres que habían sido padres en el marco de las dinámicas hospitalarias. Éstas se realizaron tras conseguir la autorización del centro clínico y el consentimiento de los informantes, asegurando en todo momento la confidencialidad de los padres mediante un código de encriptación que no permitía su identificación. Tuvieron lugar en la planta de maternidad, en concreto en la sala de lactancia, de un hospital del sur de España ${ }^{3}$ que da cobertura a 158.680 habitantes con una media de partos de 2000 nacimientos al año. Se trata de una institución caracterizada por un abordaje clínico intervencionista y productivista, como acreditan sus altos estándares de cesáreas (31\%) partos instrumentados (22\%), inducciones $\left(25 \%\right.$ ) y episiotomías $(50 \%)^{4}$. Las entrevistas se realizaron durante los primeros días postparto, justo antes del alta hospitalaria, para que el relato no fuera tamizado por el tiempo, ni perdiera su autenticidad y nitidez. Durante las mismas se siguieron las recomendaciones de Taylor y Bogdan (1987) que aconsejan tener en cuenta la necesaria privacidad que garantice una línea discursiva no fragmentada, segmentada o precodificada (Grele, 1991). La muestra se obtuvo en el año 2010, determinando el tamaño de la misma mediante el criterio de saturación, entendiendo éste como la situación a la que se llega después de haber recogido el relato de un número de informantes que garantizan la repetición de la información (D. Bertaux y I. y Bertaux, 1993). En base a estas premisas se realizaron una serie de entrevistas a veinte informantes con un duración media de treinta minutos. El foco de atención se centró fundamentalmente en tres categorías de análisis: las influencia de las dinámicas clínico-hospitalarias en la adopción de los roles parentales, los patrones de crianza y la distribución de tareas entre los padres, seleccionando los fragmentos del discurso mediante el programa de gestión de datos cualitativos MAX.QDA. Siguiendo las recomendaciones de Jociles Rubio (1999) se tuvo en cuenta la eficacia simbólica de los contextos de origen donde se producían los relatos, y en línea con lo apuntado por Jesús Ibáñez (1979), se transcribieron íntegramente siguiendo para su análisis un orden en los discursos marcado por las unidades de análisis. Éste se realizó desde un posicionamiento teórico posmoderno y de género que permitiera un reflexión profunda sobre la influencia de las rutinas hospitalarias en la adquisición de los roles parentales. En general los relatos fueron bastante

3 Por razones de confidencialidad se ha preferido mantener el anonimato del centro.

4 Datos medios referidos al periodo comprendido entre el año 2008 y 2013. 
homogéneos para cada una de las categorías de análisis, dibujando, con pequeñas discrepancias, las principales experiencias relacionadas con el fenómeno de estudio.

\section{Resultados/Discusión}

Una vez que ha nacido el bebé, comienza la fase posliminar, periodo que está cargado de simbolismos, alegorías y experiencias de aculturación. En este sentido, los testimonios de los padres muestran los dos quehaceres propios del puerperio: la recuperación física, y la instauración de los primeros patrones de crianza. Tareas que se enmarcan en el marco de una serie de rutinas y dinámicas clínicas que determinan el transcurrir de las horas, y desde las que adquieren significación ritual las experiencias posparto. A ello se suma la distribución parental de tareas, reparto mediado tanto por determinantes biológicos como por ciertas ideologías de género que se instituyen en auténticos asideros conceptuales desde los que se van construyendo los nuevos roles parentales.

\section{Construyendo el rol maternal. Encuentros y desencuentros.}

El parto se convierte en el umbral que da acceso a la maternidad, experiencia que es definida por la cultura. En este sentido, la comprensión de la liturgia posritual del parto pasa por el entendimiento previo de las nociones imperantes en cuanto a lo que socialmente significa ser "madre". Nociones que en nuestra sociedad se sitúan en la responsabilidad hacia los bebés, función básica sobre la que gira todo el ideario social, y que en general han asumido como suyo las mujeres. De esta manera, la "maternidad intensiva" (Hays, 1988) se convierte en la actividad "natural" en el puerperio, tarea que se supone que aporta gran satisfacción.

Estamos ante un ideal de maternidad satisfactoria, amorosa y feliz que se introdujo a finales del siglo XVIII, y que se presenta ahora como la respuesta natural a una idealización del proceso reproductor (Badinter, 1991). Se ha ido así construyendo la maternidad como una experiencia relajante y agradable, representación que rara vez las mujeres se arriesgan a contradecir, por lo menos "mientras esté sobre sus cabezas la espada de la culpa" (Esteban, 2000: 221). Desde estas doctrinas no se tienen en cuenta las dificultades que experimentan las mujeres durante la maternidad, y que suscitan grandes controversias entre las expectativas sociales y sus vivencias físicas y emocionales. De manera que se encuentran inmersas en una gran paradoja que las sitúa a medio camino entre las expectativas que se espera que asuman, para cumplir con las premisas socialmente establecidas, y la dura realidad del trabajo maternal, que no siempre se presenta de forma tan extraordinaria como culturalmente se entiende. Surgen así los claro-oscuros, las facetas positivas y negativas de la maternidad, que se presentan además en un momento de especial sensibilidad: «Hombre claro que hay dificultades, cuando una no sabe como hacerlo hay que aprender, tanto el bebé como la madre, sí que hay dificultades» (xxß). Se trata de un modelo único de naturalización de la maternidad que hace que la madre definida como "buena" no pueda expresar los aspectos negativos de la misma, cohibiéndola en la manifestación de sus ansiedades, conflictos, y preocupaciones (González de Chávez, 2001). Estamos, en definitiva, ante una idealización cultural que no deja ver la cara oculta de la maternidad, catadura que rara vez aparece en el tapete social ${ }^{5}$.

Las causas de esta naturalización de la crianza hay que buscarlas en las ideologías que simbolizan la función maternal como un trabajo necesario para la reproducción social (Comas D`Argemir, 2000; Díez Mintegui, 2000). Se trata de una forma de socialización que ayuda a las mujeres a interiorizar que "la tarea de ser madres va a constituir [...] su <princi-

5 En este sentido antropólogas como Juliano (1998), Imaz (2001) o Montes (2007) nos hablan de la importancia de construir metáforas culturales referidas a la maternidad que permitan recoger las tensiones, dificultades y vivencias negativas de la misma. 
pal> rasgo identitario a partir" (Blázquez, 2009: 300) del parto. Se trata de un ideario parental basado en la existencia del «instinto maternal», planteamiento desde el que se entiende la maternidad como algo "innato", y no como un aprendizaje que se tiene que ir construyendo. Se va construyendo, de esta manera, un ideario de "buena madre" que conlleva una gran carga afectiva y emocional al tener que reproducir las mujeres las expectativas sociales de cuidado. Estamos, pues, ante una construcción conceptual edificada para proteger a los bebés $^{6}$, que no tiene en cuenta las dificultades surgidas durante la crianza, se desestima así el hecho de que "incluso el amor y los sentimientos, que parecen tan espontáneos e instintivos, [pueden requerir cierto] aprendizaje” (Comas D`Argemir, 2000: 190). Realidad que afecta a todos los aspectos de la crianza, y en especial a lactancia materna, que aunque naturalizada socialmente también presenta dificultades y cierto grado de aprendizaje: «Sí, estoy intentándolo, pero me está costando mucho, pero sí que estoy intentando darle el pecho, desde el primer momento» (xxठ); «la principal dificultad es la posición, de verme yo torpe al ser la primera vez, de cómo colocar al bebé y tal» $(x x \pi)$. De esta manera, en el discurso de las madres el dar pecho no se presenta siempre como un conocimiento "innato" o "predisposición natural", por lo que en todo caso más que hablar de maternidad habría que hacerlo de maternidades (Juan, 1991).

A todo ello se suma el hecho de que la lactancia materna se muestra hoy día como lo "adecuado", visión que avalan los expertos mediante la constatación científica de toda una serie de parabienes y ventajas ${ }^{7}$. Se instaura así una coacción social y profesional en favor de que las madres lacten, olvidando que dar de mamar "es un derecho, no una obligación para las mujeres, de manera similar que otros aspectos del cuerpo y de la salud, 〈por lo〉 que exige un esfuerzo [...] para las que lo practican" (Esteban, 2000: 220). Desde estas construcciones ideológicas, la lactancia materna es predicada, a veces, como una verdadera religión, mostrándose algunos expertos, desde su autoridad institucional, como auténticos custodios inflexibles del cumplimiento de la ideología social dominante: «Fuimos a pedir un biberón, que estaba llorando, entonces nos dijeron que no, que de eso nada que de biberones nada» $(x y \lambda)$. Del discurso deviene que algunos profesionales presentan la lactancia como el único camino, como la única recomendación sanitaria, no teniendo en cuenta las situaciones y dificultades que experimentan las madres. Se produce así, en ocasiones, una auténtica fiscalización de las mujeres que no siguen el camino marcado por la doctrina oficial, responsabilizándolas de los posibles problemas que pudieran sobrevenir durante su lactancia. A ello se suma la analogía que desde determinadas representaciones culturales se ha hecho entre lactancia y afectividad, de manera que "dar de mamar «se representa como〉 estrechamente unido a la demostración de afecto y ternura" (Esteban, 2000: 221). Así, si las mujeres no se adaptan a las pautas acordadas social y profesionalmente como las "adecuadas" pueden ver cuestionado su afecto y cariño hacia sus bebés, realidad que en cierta medida coacciona sus comportamientos 8 . Se da así, a veces, la controversia de que a pesar de que "las mujeres no están enfermas, y son conscientes y responsables de sus criaturas, [en ocasiones] no se considera su voluntad ni criterio para tomar decisiones mientras permanezcan en el

6 En este sentido, autoras como la filósofa francesa Badinter (1991), o las antropólogas españolas como Osorio (1999; 2001), Montes (2007) y Blázquez (2009), defienden que la maternidad depende de la cultura y no de algo "natural" o "innato".

7 Entre los beneficios para la madre expuestos por los expertos se encuentran una mejora de la involución uterina, un menor riesgo de enfermedades como diabetes, cáncer de pecho y ovario y una menor depresión materna. Las principales ventajas para el niño son un menor riesgo de enfermedades como otitis, gastroenteritis, infecciones respiratorias, dermatitis, asma, obesidad, diabetes, leucemia, síndrome de muerte súbita y enterocolitis. (Gómez García, 1999; Calzado et al., 2000; Díaz et al., 2006).

8 La profesora de psicología de la Universidad de Pennsylvania Diane E. Eyer (1995) afirma que detrás de estas políticas de promoción de lactancia materna se encuentra el protagonismo de los expertos, que mediante la definición sanitaria de las pautas de la maternidad controlan la vida familiar y acentúan su poder. 
hospital, si no es contrastando con la voluntad del personal sanitario" (Montes, 2007: 320). Ello genera ciertas inseguridades y ansiedades maternas que puede llegar a culpabilizarlas: «le hemos dado chupete y ya no sé, tengo la manía que no sé si por el chupete le cuesta ahora engancharse» $(x x \mu)$. En este sentido, la presión del entorno puede, a veces, inducir a la adquisición de ciertos remordimientos e inducidos, en parte, por la propia matriz social preexistente.

Estamos ante una construcción cultural de la maternidad que las mujeres, como miembros de la misma colectividad, interiorizan, intentando reproducir en sus tareas de crianza los discursos hegemónicos. Desde esta asunción la mayoría de las madres inician la lactancia materna exclusiva durante su instancia en las maternidades (Calvo et al., 1992): «pecho le estoy dando, y mientras pueda pues le estaré dando pecho, claro, si eso es bueno para el niño también lo es para mí» (xxx); «sí solo pecho [...] estoy dándole a demanda, si me pide a las tres horas como si me pide a la hora» $(x x \pi)$. Y con el comienzo de la lactancia, $\mathrm{y}$ a pesar de las campañas de naturalización en favor de la misma, comienzan las dificultades. En primer lugar se encuentran las molestias derivadas del duro ritual iniciático intervencionista vivido en paritorio, molestias que, a veces, dificultan la «maternidad intensiva»: «me siento muy molesta, no me puedo mover bien [...] no me veo cómoda para colocar al niño al pecho» $(x x \omega)$. Y es que el paso hacia la maternidad, a través de la liturgia hospitalaria, deja con frecuencia toda una serie de limitaciones físicas que obstaculizan las tareas de lactancia materna: «el problema es que al tener la cesárea no me puedo incorporar bien [...] me cuesta enganchármelo por que no me puedo poner de lado» (xxy). A ello se une la desazón adicional que produce, en caso de cesárea, el retardo en el inicio de la lactancia materna, que no se instaura hasta las dos o seis horas postparto (Pérez et al., 2003). Realidad que algunas madres relatan con ansiedad, conscientes, desde la interiorización a los discursos profesionales ${ }^{9}$, que ello puede dificultar la instauración de la lactancia ${ }^{10}:$ «por eso me dio tanto coraje eso de la anestesia de la cesárea porque tardaron mucho tiempo en bajarme y no pude engancharme al crío nada más nacer como yo quería [...] tres horas y media o casi cuatro, yo quería que hubiera sido antes, me hubiera gustado ponerlo antes al pecho» (xxठ). Los desasosiegos, e incertidumbres surgidos a las neofitas durante la asunción del nuevo rol de madre, sustancian, pues, parte de las representaciones típicas de esta vivencia existencial.

A todo lo apuntado hasta ahora, hay que sumar ciertas contradicciones que con frecuencia surgen entre las diferentes ideologías sociales y profesionales sobre la lactancia materna, y la propia dinámica clínica que en ocasiones no ayuda a su instauración. En este sentido, en ocasiones se instaura un circuito asistencial impregnado de nociones productivistas que, desde interpretaciones de coste-eficacia, priorizan disminuir la asistencia durante el puerperio para que la mujer autogestione su salud: "no nos han dicho nada, aquí es que lo único que nos preguntan le habéis dado el pecho, ha hecho caca y poco más» (xxభ). En el marco de estas representaciones se produce una disminución de la atención en un momento de especial vulnerabilidad para las madres, el puerperio, al considerar que las principales situaciones de riesgo, el embarazo y el parto, ya han concluido: «no te ayudan lo suficiente aquí, no veo yo la ayuda para lactancia materna [...] varias veces hemos preguntado a las enfermeras y les hemos dicho que el niño no se engancha al pecho [...] y tampoco te vienen aquí a ayudarte» ( $x x \delta$ ). De los relatos maternos subyace cierta filosofía fabril centrada en la consecución de un bebé sano, principal bien social, conseguido éste parece que se pierde

\footnotetext{
9 El obstetra francés Michael Odent (1990) ha expuesto las ventajas de la instauración temprana del vinculo madre-hijo y del inicio precoz de la lactancia materna. Convirtiéndose ésta en la doctrina oficial que recogen las diferentes estrategias de asistencia al parto de los diferentes organismos oficiales (MSC, 2007; MSPS, 2010). $10 \mathrm{La}$ evidencia científica muestra que la postergación del contacto piel con piel durante la primera toma, junto con el uso de biberones, son las principales causas de abandono de la lactancia materna (Otal et al.., 2012; Hernández et al.., 1988).
} 
cierto interés clínico por las mujeres. Esta falta de interés por las dificultades del posparto contribuye también a mantener "la tradicional representación de la maternidad como un sacrificio o sufrimiento, [...] precio a pagar por la naturaleza sexual" (Montes, 2007: 324): «a mí no me asesoraron en ningún momento, lo que sé es [...] lo que haya podido leer y por el instinto maternal ni más ni menos, pero no en ningún momento te dicen nada allí, en ningún momento» $(x x x)$. Parece que se trata de una cierta desatención asistencial desde la que se ignoran las ventajas que supone el asesoramiento sanitario en materia de lactancia, consejo que mejora tanto el inicio como el mantenimiento de la misma (Blanco, Burgos y Ruiz, 1999). Se conforma así con frecuencia una red social que, aunque se envista de apoyo, cumple sobre todo una misión de control social. Da la impresión de que los sanitarios se limitaran a controlar la conducta materna en favor de la lactancia, mientras obvian, desde un enfoque productivista, la ayuda y asesoramiento que necesitan en estos momentos de incertidumbre: «nada, le han dicho que se lo ponga al pecho pero no han venido y le han puesto al crío, oye que esto es así, esto se pone así, nos han dicho nada [...] no han llegado $y$ lo han puesto al crío, esto intenta hacerlo así, intenta hacerlo de esta manera» (xyభ). A ello se suman las contradicciones que muestran los propios profesionales, en cuanto a sus perspectivas sobre la lactancia, ya que en ocasiones ha sido impuesta sin que se consensuen las posiciones oficiales, por lo que surgen ciertas paradojas asistenciales: «mira, desde que he bajado aqui a planta cada una te dice una cosa, unos te dicen que sí, otros que no, otros se encogen los hombros como tú haz lo que tú quieres que es tuyo» (xxu). En este contexto de la desorientación algunos padres acaban aferrándose a cualquier recurso disponible: «te ayudan más las personas no cualificadas, como celadores, que las personas altamente cualificadas [...] no hay una persona especializada en venir y decir esto se hace así» (xyठ). El desinterés mostrado por algunos profesionales, lleva a muchas madres a cuestionar la propia formación de los expertos en temas de lactancia (Corrêa y julián, 2002; Andreo y Silvan, 2004): «yo creo que la gran mayoría no <están formados [...] que es como un poco la experiencia, que la gran mayoría son madres, y lo que ellas han hecho o que tal, pero tampoco ni te aconsejan para una cosa, ni te aconsejan para otra, es como que es tuyo haz lo que quieras [...] te dicen si es que todavía acaba de nacer, eso es normal, pero eso no son explicaciones lógicas porque eso lo sabemos todos» $(x x \mu)$.

Los testimonios muestran la realidad de una situación que, en ocasiones, pone a prueba los recursos maternos. Las mujeres, inducidas por la presión social, e inmiscuidas en las dificultades propias del proceso, son a veces llevadas a una zona marginal en sus expectativas. La experiencia materna se va así tamizada con el sufrimiento, y con toda una serie de experiencias de inseguridad sazonadas, a su vez, con dificultades, sensaciones molestas y lesiones físicas. En este pulmento afloran con frecuencia toda un serie de inseguridades sobre el correcto funcionamiento de la propia biología ${ }^{11}$ : «no me ha dado la subida, yo es que no sé los motivos, no sé si es por haberme puesto la anestesia que paso mucho tiempo hasta que le puede dar y al crío le dieron primero biberón» $(x x \psi)$; «tengo miedo de que mi pecho no dé la leche cuando lo necesite, y de que el crío pierda peso y tenga que abandonar la lactancia $y$ darle un biberón» $(x x \omega)$. A ello se suman las molestias derivadas de la lactancia, que, a veces se convierte en una tarea ardua, monótona y solitaria, a la vez que inhabilita, en cierta medida, para el desempeño de otros roles sociales. En este caldo de cultivo algunas mujeres se cuestionan si merece la pena continuar con el gran esfuerzo que supone la instauración y mantenimiento de la misma, surgiendo así dudas sobre si se podrá mantener la misma: «quizás abandonaría la lactancia por desesperación mía [...] más que por el bebé, creo que [...] sería por mí, porque me desesperaría» $(x x \delta)$. El resultado de este círculo vicioso de

11 Los estudios coinciden en señalar que estas inseguridades, entre las que se encuentra el miedo a no producir suficiente leche o a que el bebé no gane suficiente peso, son uno de los motivos principales para el abandono de la lactancia materna (Bustos et al, 1999; Gómez-Galán y López, 2003) 
ansiedades, frustraciones y molestias es, en muchos casos, el abandono total o parcial de la lactancia materna exclusiva, tal como muestra el testimonio de algunas madres: ${ }^{12}$ : «anoche mismo se quedó con hambre después de darle biberón, y esta madrugada como ya le ha-

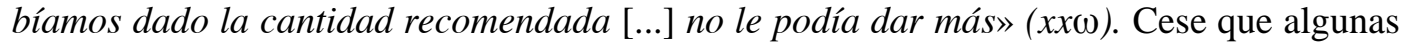
mujeres necesitan justificar ante los expertos, seguramente como resultado de las ideologías sociales que naturalizan la lactancia materna como la conducta "normal": «pues si es que yo lo intento ponerlo, pero no me da subida y los tengo muy blandos» (xx $\psi)$; «el crío ha pesado bastante poquillo y no está fuerte para tirar del pecho, que yo quisiera darle pecho, pero claro, estoy con el miedo de que pierda peso» ( $x x \omega)$. Se instaura así en el ideario colectivo de las madres cierta tensión psicológica, circunstancia motivada tanto por el abandono institucional como por los esfuerzos por mantener el orden social establecido.

En el marco de esta configuración clínica los padres van configurando su propio estilo de crianza, patrón que en línea con las filosofías productivistas y presiones de fondo preexistentes dirigen, en cierta medida, hacia la adopción de modelos distantes ${ }^{13}$. Se produce, así, cierta paradoja entre las doctrinas oficiales de apego y las propias dinámicas hospitalarias que, en consecuencia con las nociones tecnocráticas cosmopolitas, dificultan el contexto necesario para su realización. Se van conformando así, en ocasiones, las tres características propias del patrón de crianza distante: la instauración de la lactancia artificial, consecuencia del abandono institucional y de las propias dificultades en el aprendizaje, la ausencia de colecho, bajo las ideologías de la independencia precoz: «él tiene que dormir en su cuna entiendes, y nos va a costar acostumbrarlo» $(x y \lambda)$, y la tendencia social a no auxiliar los llantos del bebé, bajo el pretexto de que los bebés no se acostumbren: «todo el mundo aconseja: anda... [...] si cada vez que llore, como lo cojas y se quede en los brazos, lo acostumbras a los brazos [...] el no cogerlo es por ese miedo a que se acostumbre» (xy $\mu)$. En este sentido, parecería que los nuevos miembros de la sociedad tecnológica fueran dirigidos, desde su tierna infancia, a interiorizar las premisas básicas que garantizan la reproducción del sistema productivista, entre la que se encuentra la autonomía y la independencia precoz.

Tomando como base los relatos, se podría concluir que las filosofías institucionales oficiales que naturalizan de la crianza y fomentan culturas de apego no son más que una estrategia superficial, que se desmorona al chocar con las características de fondo de las dinámicas asistenciales características del parto hospitalario. Se trata más bien de un consenso social, de una especie de «hipocresía oficial», desde la que es muy difícil que se implante un auténtico modelo de apego, mientras el proceso esté vertebrado por las nociones posthumanistas y productivistas. De esta manera, parece que estamos ante una experiencia puerperal incongruente en la que se presiona socialmente a las mujeres para que instauren patrones naturales de crianza, a la vez que las propias limitaciones sufridas tras la dura liturgia del nacimiento en paritorio, y el déficit asistencial por parte del personal, las dirige hacia un patrón de crianza distante. Desde esta contradicción, es normal que las madres manifiesten haber experimentado muchas dificultades, apareciendo durante este primer contacto con su nuevo rol maternal muchas insatisfacciones, frustraciones. Conflictos que seguramente persistirán hasta que se adapten a la nueva situación y "aprendan" a desempeñar su nuevo rol.

\section{El papel de los padres. Distribuyendo tareas: de la intencionalidad a los hechos}

La maternidad/paternidad, y las consecuencias culturales que se derivan de ella, "son

12 Las investigaciones muestran como las madres, a pesar de iniciar la lactancia materna en el hospital, la abandonan en los primeros días. Ello hace que la duración promedio de la misma sea muy baja, entre ocho y diez semanas, frente las 24 semanas que recomienda la OMS (Gómez et al.., 1997; Barriuso et al.., 1999)

$13 \mathrm{La}$ antropóloga americana Meredith Small (1999) afirma que desde la implantación del modelo de crianza distante no se respetan las necesidades innatas de apego que tienen los bebés, cuyo llanto, a pesar de ser interpretado en nuestra sociedad como algo molesto, es simplemente una señal de alarma. 
una construcción cultural enmarcada en las relaciones de género, desde las que se distribuye de forma diferenciada las tareas y responsabilidades de mujeres y hombres en el cuidado de las criaturas." (Montes, 2007: 314). Esta consideración es vital para comprender la forma en que los padres reparten las tareas durante el puerperio hospitalario, ya que es precisamente a partir esta distribución cultural de roles desde la que se posiciona y cobra sentido la distribución de las labores de crianza.

En los últimos años, y desde la difusión de políticas en favor de la igualdad entre sexos, se han publicado diferentes estrategias, textos y leyes en defensa de la participación de los hombres en los procesos reproductivos y de crianza ${ }^{14}$, documentos auspiciados por toda una serie de efectos positivos que hipotéticamente supondría su implicación (Osborne, 1993; Horvath Cea, 1999). Todo ello ha conformado un frente en favor de la paulatina democratización de la institución familiar (Alberdi, 1999), cuyo objetivo es involucrar a los padres como corresponsales en las labores de crianza de sus hijos. Influidos por estas ideologías culturales, se ha producido en los últimos años una mayor intervención de los padres en las labores domésticas (Meil, 2005), sobre todo en aquellas tareas cotidianas que muestran mayor flexibilidad, entre las que se incluyen las tareas de crianza (Carrasquer et al., 1998). Estamos asistiendo, de esta manera, a una paulatina implicación de los hombres en temas que tradicionalmente habían sido considerados como maternales, hablándose en este sentido de «maternidad compartida» y «paternidad responsable». Esta actitud se observa ya desde el mismo nacimiento de los bebés, mostrándose los padres colaboradores durante el puerperio hospitalario: «yo lo que haga falta, aqui darle, limpiarlo, levantarlo, que no llore sobre todo [...] que no llore y estar ahí pendiente de él, lo que sea» (xyx); «de todo, cambiarle los pañales, ponérselo, cogerlo de la cuna para que le dé pecho, ponerlo en la cunita cuando se duerme, está dando el pecho y terminar de comer se queda dormido, lo cojo lo pongo en la cuna otra vez, le cambio de pañal [...] todo la baño, todo». Sus labores de crianza se producen además en el momento en que las madres más lo necesitan, si tenemos en cuenta que se encuentran en plena recuperación del parto y en el marco de cierto desinterés asistencial por el puerperio: "yo esto ahí ayudándola porque ella no se puede levantar, no se puede ni

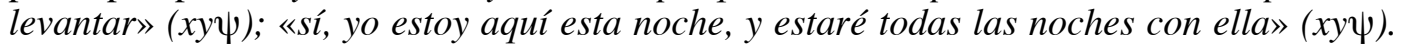
Circunstancia que alaban las madres, y que sin duda es útil en un momento de contradicciones y gran carga emocional: «todo, lo hace muy bien, él lo hace muy bien» (xxx); «todo si me lo hace él todo» $(x x \boldsymbol{\alpha})$. Los padres se envisten, de esta manera, en personas recurso que salen al auxilio de la encrucijada emocional de las madres.

Sin embargo, la forma en que se establecen las cargas de crianza denota todavía ciertas desigualdades ${ }^{15}$. En este sentido, las tareas de crianza en los padres se presentan todavía como una opción, e incluso como un derecho, pero rara vez como una obligación. Desde estas nociones el padre se presenta como "colaborador" pero no como corresponsable de la crianza, recayendo así el rol de cuidadora principal en la madre: «que sí, que sí, yo intento colaborar en todo lo que puedo» $(x y \omega)$. Realidad mediada, en parte, por la propia naturaleza lactadota de la mujer que hace patente las limitaciones de la biología masculina naturalizando, así, la crianza como una labor femenina: «yo de todo, menos darle pecho de todo [...] no le puedo dar pecho porque no tengo si no» (xyл). Representación que interiorizan las

14 Entre estos documentos se encuentran el texto final de la Conferencia Internacional de Naciones Unidas sobre población y desarrollo de 1994, que señala la necesidad de desarrollar estrategias para activar la participación de los hombres en las tareas de crianza, o el documento en favor de la participación de los hombres en la salud sexual y reproductiva de UNICEF de 1998. A nivel nacional destacan la Ley de Igualdad de 2007, y el Plan Estratégico Nacional de Igualdad de Oportunidades (2008-2011), en los que se enfatiza la necesidad de corresponsabilizar a los hombres para superar la división sexual otorgando permisos como el de paternidad.

15 Numerosos estudios coinciden en la persistencia de desigualdades de género en cuanto a la distribución del trabajo doméstico y de crianza, presentándose la maternidad compartida como una realidad minoritaria (Pedrero, 2003; Larrañaga et al., 2004; Díez Mintegui, 2000) 
madres ${ }^{16}$ desde la asunción "natural" de este rol de cuidadora principal, mostrando serias dudas sobre una implicación duradera de sus parejas: "sí colabora, de momento sí, de aquí

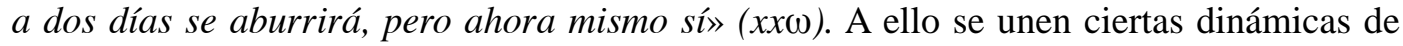
exclusión del propio circuito asistencial que hace que los padres tengan que abandonar a las madres durante las visitas médicas, dejando solas a sus mujeres en un momento marcado por las limitaciones físicas.

Nos encontramos, pues, ante una situación ambivalente en la que si bien los padres se muestran disponibles durante el puerperio hospitalario, constituyendo el principal apoyo de las madres, la corresponsabilidad total encuentra sus limitaciones en una distribución de roles mediada por las capacidades de lactación propias de la naturaleza materna. En este sentido, los padres construyen su rol parentental en oposición a las madres, desde la premisa de sujeto "colaborador", mientras las madres ostentan el rol de cuidadoras principales. La configuración social de la lactancia materna como la conducta "correcta" afianza esta distribución de roles determinada biológicamente, sirviendo de mediadora en la reproducción humana entendida como bien social.

\section{A modo de conclusión}

En los contextos occidentales el contacto con las primeras asignaciones de rol parenteal se produce ordinariamente en el marco de las dinámicas asistenciales propias de las instituciones hospitalarias. En este «habitus» se va dibujando lo que significa ser padres, realidad que tras el umbral del parto se prefigura a través de las dos tareas típicas del puerperio: la recuperación materna y la instauración de las labores de crianza. De esta manera las primeras atribuciones de rol se producen en el seno de una serie de decisiones que definirán el modelo de crianza, patrón que cobra vida a través de las actitudes ante realidades como el tipo de alimentación, la forma de dormir o la conducta ante los llantos del bebé. En este sentido, las madres se encuentran con la dificultad de una presión social que ha situado el rol de la "buena madre" en las actitudes de apego que incluyen la lactancia materna exclusiva. Coerción que experimentan a la vez que son sometidas a una dinámica asistencial que, desde nociones tayloristas, quita importancia a las tareas de auxilio profesional durante el puerperio. Se prefiguran así toda una serie de frustraciones en la asignación del nuevo rol maternal, inducidas, en parte, por las propias dinámicas clínicas productivistas que no consideran que interese reinvertir tiempo y dinero una vez que se ha obtenido el producto final, el bebé. Pese a ello, a las madres les queda la dura tarea de recuperarse, y lo que es más importante, cuidar a sus bebés, trabajo que necesita de un proceso de aprendizaje y asesoramiento, pese a que haya sido simbolizado culturalmente como algo «innato». Se van configurando así los claro-oscuros de una asignación del rol maternal impregnado de sentimientos de soledad e impotencia. En estas circunstancias el recurso más cercano es el padre, que configura su rol parental en oposición al de las madres, instaurándose así en un sujeto "colaborador" cuyas tareas de auxilio sirven paradójicamente para reafirmar el rol materno de cuidadora principal. Realidad que se prefigura desde unas coordenadas sociales que sitúan la conducta deseable en la instauración de una lactancia materna exclusiva que hace patente, y en cierta medida inevitable, la asignación de roles parentales desde las propias características de la biología materna.

\footnotetext{
16 El obstetra francés Bernad This (1982) nos aporta una interesante revisión sobre las conductas tenidas por maternales en los machos de especies animales, y por los relatos mitológicos que hablan de los embarazos y partos masculinos. Su exploración etológica y mitológica llega a la conclusión de que existe una inhibición social del deseo materno en los hombres.
} 


\section{Bibliografía}

Alberdi, Inés (1999). La nueva familia española. Madrid: Taurus Ediciones.

Andreo, S.O y Silvan, C.G. (2004). «Las dificultades maternas en el proceso de amamantamiento materno de prematuros en una UCI neonatal». Rev. Latino-am Enfermagem, 12(4), 597605

Badinter, Elisabeth (1991). ¿Existe el instinto maternal? Historia del amor maternal. Siglos XVII al $X X$. Barcelona: Paidós.

Barriuso, L.M ; Sánchez-Valverde, F; Romero, C.; et al. (1999). «Epidemiología de la lactancia materna en el centro-norte de España». Anales españoles de pediatría, 50 (3), 237-243.

Bertaux, Daniele y Bertaux, Isabelle (1993). «Historias de vida del oficio de panadero», en J.M. Marianas y C. Santamaría (eds.), La historia oral: Métodos y experiencias. Madrid: Debate, 231-251.

Blanco, A.I; Burgos, L. y Ruiz, M.C. (1999). «Influencia de la educación sanitaria en la elección de la lactancia materna a puérperas, como mejor opción para la alimentación de sus hijos». Revista de Enfermería Clínica, 9(5), 194-199.

Blázquez, Ma Isabel (2009). Ideologías y prácticas de género en la atención sanitaria del embarazo, parto y puerperio: el caso del área 12 de la Comunidad de Madrid. Tesis Doctoral. Departamento de Antropología, Filosofía y Trabajo Social. Universidad d Rovira i Virgili.

Bustos, Rafael; Sánchez, María Guadalupe; Ruiz, Lidia; et al. (1999). «Costumbres en el otorgamiento de la lactancia materna por parte del personal de enfermería». Revista de Enfermería del Instituto Mexicano de Seguridad Social, 7(1), 33-36.

Calvo, B; Millán, C; Álvarez, J.D.; et al. (1992). «Actitud materna ante la lactancia natural y dificultades en el puerperio inmediato». Revista Atención Primaria, 10(3), 650-654.

Calzado, Miriam; Rodríquez, Loida; Vargas, Eresmilda.; et al. (2000). «Influencia de la lactancia materna en la salud del niño». Revista Cubana de Enfermería, 16(2), 122-127.

Carrasquer, Pilar; Torns, Teresa; Tejero, Elisabet; et al. (1998). «El trabajo reproductivo». Papers: Revista de Sociología, 55: 95-114.

Comas D`Argemir, Dolors (1996). «Economía, cultura y cambio social». En: Prat y Martínez (eds.), Ensayos de Antropología Cultural. Barcelona: Ariel, 104-113.

Comas D`Argemir, Dolors (2000). «Mujeres, familia y estado del bienestar». En: Del Valle (ed.), Perspectivas feministas desde la antropología social. Barcelona: Ariel, 187-204.

Corrëa, C.R. y Juliani, C.M. (2002). «Lactancia materna: conocimiento y actitudes del equipo de enfermería». Revista Paulista Enfermagem, 21(1), 84-94.

Díaz Gómez, Marta; Gómez, Carmen Isabel. y Ruiz, Ma Jesús (2006). Tratado de enfermería de la infancia y la adolescencia. Madrid: McGraw-Hill Internacional.

Diaz Gómez, N.M; Hernández, C; Díaz Gómez, J.M; et al. (1988). «Factores relacionados con la lactancia materna en nuestro medio». Revista de Enfermería Científica, 70, 19-25.

Díez Mintegui, María del Carmen (2000). «Maternidad y orden social. Vivencias del cambio». En: Del Valle (ed.), Perspectivas feministas desde la Antropología social. Barcelona: Ariel, 155-185.

Esteban, María Luz (2000). «La maternidad como cultura. Algunas cuestiones sobre lactancia materna». En: Perdiguero y Comelles, Medicina y cultura. Estudios entre antropología y medicina. Barcelona: Bellaterra, 207-226.

Eyer, Diane. E. (1995). Vinculación madre-hijo. Una ficción científica. Barcelona: Herder.

González de Chávez, Asunción (2001). «Preparación para el parto versus preparación para la maternidad y paternidad», en Género y salud. Madrid: Instituto de la mujer, 24-23.

Gómez García, Carmen Isabel (1999). Promoción de la lactancia materna: una propuesta de intervención educativa. Departamento de teoría e historia de la educación. Tesis doctoral. Universidad de Murcia.

Gómez-Galán, Rafael y López, Ma José (2003). «Lactancia natural en el área de salud de Mérida. Incidencia, duración y tendencias actuales». Revista de Enfermería Científica, 256-257, 57-62.

Gómez, M; Baeza, J.E; González, J.A.; et al.(1997). «Prevalencia y características de la lactancia materna en el medio rural». Revista de. Atención Primaria, 19(6), 318-322. 
Grele, Ronald J. (1991). «La historia y sus lenguajes en la entrevista oral, quién contesta a las preguntas de quién y por qué». Historia y Fuente oral, 5, 106-127.

Hays, Sharon (1988). Las contradicciones culturales de la maternidad. Barcelona: Paidós.

Hernández Garre, José Manuel (2011). El parto hospitalario e intervencionista. Una construcción social de la maternidad. Tesis doctoral. Departamento de Filosofía. Universidad de Murcia.

Hernández Garre, José Manuel y Echevarría Paloma (2014). «La parte negada del parto institucionalizado. Explorando sus bases antropológicas». Revista de dialectología y tradiciones populares, 69 (2), 327-348.

Horvath Cea, Cristina (1999). «La importancia del padre -antes, durante y después del nacimiento de su hijo/a (Observaciones desde el ámbito de la preparación al parto psicoprofiláctica). Tú serás el padre de mi hijo». En: González Chávez (comp.), Subjetividad y ciclos vitales de las mujeres. Madrid: Siglo XXI, 87-110.

Ibañez, Jesús (1979). «Interpretación y análisis del discurso». En: I. Jesús, Más allá de la sociología. El grupo de discusión, técnica y crítica. Madrid: Siglo XXI, 333-351.

Imaz, Elixabete (2001). «Mujeres gestantes, madres en gestación: Metáforas de un cuerpo fronterizo». Política y Sociedad, 36, 97-111.

Jociles, Ma Isabel (1999). «Las técnicas de investigación en antropología». Gazeta de Antropología, $15,15-01$

Jordan, Brigitte (1993). Birth in four cultures. A Crosscultural Investigation of Childbirth in Yucatan, Holland, Sweden and the United States. Montreal: Eden Press Women's Publications.

Juan, Montse (1991). «Crear el nacimiento: la medicalización de los conflictos en la reproducción». Revista de Investigaciones Sociológicas, 53, 29-51.

Juliano, Dolores (1998). Las que saben. Subculturas de las mujeres. Madrid: Horas y Horas.

Larrañaga, Isabel; Arregui, Begoña. Y Arpal, Jesús (2004). «El trabajo reproductivo o doméstico», Gaceta sanitaria, 18 (1), 31-37.

Menéndez, Eduardo, L. (2000). «Factores culturales. De las definiciones a los usos específicos». En: Perdiguero y Comelles (eds.), Medicina y Cultura. Estudios entre la Antropología y la Medicina. Barcelona: Bellaterra, 163-188.

Menéndez, Eduardo. L. y Di Pardo, Renée B. (1996). De algunos alcoholismos y algunos saberes. Atención primaria y proceso de alcoholización. México: Casa Chata.

Meil, Gerardo (2005). «La redefinición de la división del trabajo doméstico en la nueva familia urbana española». Revista de Investigaciones Sociológicas, 80, 69-94.

Ministerio de Sanidad y Consumo (2007). Estrategia para la atención al parto normal. Madrid: Autor.

Ministerio de Sanidad y Política Social (2010). Estrategia Nacional de Salud Sexual y Reproductiva. Madrid: Autor.

Montes, Ma Jesús (2007). Las culturas del nacimiento. Representaciones y prácticas de las mujeres gestantes, comadronas y médicos. Tesis doctoral. Departamento de Filosofía, Antropología y Trabajo Social. Univesitat Rovira i Virgili.

Odent, Michael (1990). El bebé es un mamífero. Madrid: Mandala.

Osborne, Raquel (1993). La construcción sexual de la realidad. Madrid: Cátedra.

Osorio, Rosa María. (1999). «Antropología del género y Antropología médica: convergencias posibles y necesarias». Ankulegi. gizarte antropología aldizkaria., 63-70.

Osorio, Rosa María (2001). Entender y atender la enfermedad. Los saberes maternos frente a los padecimientos infantiles. México: CIESAS, INAH, INI.

Otal-Lopaus, Silvia; Morera- Liánez, Leticia; Bernal-Montañes, Ma José; et al. (2012). «El contacto precoz y su importancia en la lactancia materna frente a la cesárea». Matronas Profesión, 13(1), 3-8.

Pedrero, Mercedes (2003). Distribución del tiempo entre trabajo domestico y extra-doméstico según la posición en la familia. Consulta técnica sobre contabilización de la producción no remunerada de servicios de salud en el hogar (Ponencia), Washington D.C., 4-5 Diciembre, (paper).

Pizzini, Franca (1989). «The expectant mother as patient: a research study in Italian matermity wards». Health Promotión. Oxford University Press, 4 (1), 1-10. 
Ranisio, Gianfranca (1998). Venire al mondo. Credenze, pratiche, rituali del parto. Roma: Meltemi. Rodríguez, R. (2015). «Aproximación antropológica a la lactancia materna». Revista de Antropología Experimental, 15, 407-429.

Romani, Oriol (1992). «Marginación y drogodependencias. Reflexiones en torno a un caso de investigación». En: Álvarez Uría (Comp.), Marginación e inserción. Los nuevos retos de las políticas sociales. Madrid. Eudymión, 259-281.

Pérez, Isabel María; Romero, Manuel Enrique; Nuñez, Esperanza; et al. (2003). «Prevalencia de la lactancia materna y práctica hospitalaria en las Maternidades de Sevilla». Revista Metas de Enfermería, 6 (59), 18-23.

Small, Meredith (1999). Nuestros hijos y nosotros. Barcelona: Ed. Vergara.

Stoller, Nancy (1974). Forced labor: maternity care in the United States. Oxford: Pergamon studies in critical sociology.

Taylor, S.J. y Bogdan, R. (1987). Introducción a los métodos cualitativos de investigación. Barcelona: Paidós.

This, Bernard (1982). El padre: acto de nacimiento. Barcelona: Paidós.

\section{3}

